\title{
L'économie sociale : un troisième outil stratégique dans le coffre du développeur
}

\author{
R. Mathieu Vigneault \\ CLD de la région d'Acton
}

D epuis plusieurs décennies, l'économie sociale fait partie des façons de faire des Québécois. Les Caisses populaires, les coopératives alimentaires et de services à domicile, les Offices municipaux d'habitation $(\mathrm{OMH})$, etc. ne sont que quelques exemples de ce que la solidarité et la prise en charge de la communauté par elle-même a pu engendrer dans le domaine économique par le passé ${ }^{1}$.

Après quelques années éprouvantes au cours du règne de l'économie libérale, elle est réhabilitée lors du Sommet sur l'économie et l'emploi de 1996. Aujourd'hui plus que jamais, l'économie sociale est partie intégrante du modèle québécois, soit une économie plurielle qui accorde une place importante à l'intervention de l'État, mais qui s'appuie aussi sur un imposant réseau d'entreprises privées et collectives.

À vrai dire, l'économie sociale, est devenue un incontournable de l'économie québécoise aux côtés de l'économie libérale (entreprises privées) et de l'économie publique (sociétés d'États) ${ }^{2}$. Dans cet esprit, elle constitue un outil privilégié de développement

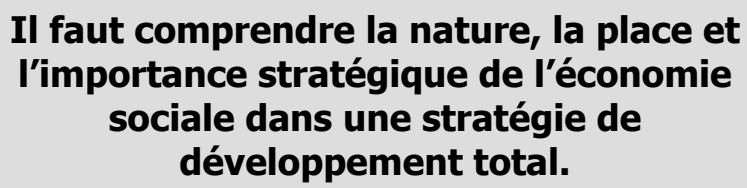

stratégie de développement total et, plus important encore, de gérer cet outil comme il se doit, c'est-àdire en évitant de confondre projet communautaire de même que cadeau politique avec véritable économie sociale.

\section{Une économie plurielle}

Tous plus importants les uns que les autres, trois éléments fondamentaux composent le modèle économique québécois. Il s'agit, au premier chef, d'une économie dite publique, soit celle de l'égalité des chances et de l'accès pour tous à des conditions de vie équitables. Toutefois, l'État québécois s'est grandement transformé depuis la Révolution tranquille. D’État promoteur et entrepreneur de grands projets de développement dans les années 1960-1970, il est devenu un État partenaire. Cela implique donc que les fonds publics sont aujourd'hui plus souvent en aval qu'en amont du développement. Il n'en demeure pas moins que l'économie publique est encore omniprésente et que l'État, s'il est de moins en moins promoteur, représente toujours un partenaire privilégié de développement. socio-économique pour l'ensemble du Québec, mais peut-être encore davantage pour les régions périphériques du Québec. À cet égard, l'économie sociale représente une voie non négligeable pour les organismes de développement tels que les CLD (Centres locaux de développement) et les SADC (Sociétés d'aide au développement des collectivités), afin de stabiliser et de solidifier le tissu socio-économique de leur région.

Toutefois, et c'est le principal propos de cet article, encore faut-il comprendre la nature, la place et l'importance stratégique de l'économie sociale dans une
Pour sa part, l'économie libérale est celle de la croissance économique. Celle qui autorise chaque personne à espérer mieux et à bâtir un patrimoine individuel spécifique. Cependant, étant assujettie aux lois du capitalisme et, qui plus est, étant particulièrement sensible aux nouvelles règles imposées par la globalisation de la production et de la mondialisation des marchés, l'économie libérale est plus que jamais un géant au pied d'argile. Si, dans certains cas, une économie libérale diversifiée peut propulser un territoire ou une région vers la prospérité, plusieurs exemples récents ont démontré qu'elle peut aussi la mener rapidement vers la précarité. Pensons à l'in- 
dustrie du textile dans l'ouest de la Montérégie et au sud de la Beauce, à l'industrie du bois d'œuvre au Témiscouata, à l'industrie minière en Estrie, Murdockville et Thetford Mines, etc. Ceci dit, lorsque diversifiée, l'économie libérale constitue la locomotive de l'enrichissement et du développement d'un territoire. Il s'agit d'un incontournable qui, cependant, ne devrait pas œuvrer seul, au risque de fragiliser irrémédiablement la roue du développement durable.

De son coté, l'économie sociale est celle du développement à visage humain et de l'enrichissement solidaire et éthique des communautés. L'économie sociale tire son sens d'activités économiques inspirées par la notion de services à la collectivité et de réinvestissement dans celle-ci. L'économie sociale est un type d'économie par la communauté afin de combler certains besoins de celle-ci. Elle ne risque donc pas d'être relocalisée là où la main-d'œuvre est moins coûteuse ou les matières premières plus abordables. Ainsi, l'économie sociale ne poursuit pas l'objectif de la profitabilité économique, mais plutôt d'une certaine viabilité financière doublée d'une importante profitabilité sociale.

\section{Une économie... sociale ?}

Selon les gens du Chantier de l'économie sociale, on qualifie d'économie sociale les entreprises ou activités qui font la promotion des valeurs suivantes :

- service aux membres ou à la collectivité;

- autonomie de gestion;

- démocratie (impliquant usagers et travailleurs);

- primauté des personnes et du travail sur le capital;

- participation (prise en charge et responsabilité individuelle et collective).

Bien entendu, il semble que ce concept combine à la fois deux notions qui sont fréquemment placées en opposition, soit «économie ", qui renvoie à la production concrète de biens ou de services ayant l'entreprise comme forme d'organisation et contribuant à une augmentation nette de la richesse, et "sociale », qui réfère à la rentabilité sociale et non purement économique d'activités, c'est-à-dire au profit non tangible qu'une communauté peut retirer d'un projet. Cette rentabilité s'évalue entre autres au niveau de la contribution au développement démocratique, par le soutien à une citoyenneté active, par la promotion de valeurs et d'initiatives de prise en charge individuelle

et collective. La rentabilité sociale contribue par conséquent à l'amélioration de la qualité de vie et du bien-être de la population, notamment par l'offre d'un plus grand nombre de services abordables ${ }^{3}$.

Par ailleurs, si l'efficacité caractérise l'économie privée au même titre que la redistribution qualifie l'économie publique, l'économie sociale favorise entre autres la démocratie par la présence d'usagers et de professionnels dans les lieux décisionnels ${ }^{4}$. À titre d'exemple, à la suite de l'adoption de la loi 34, plusieurs CLD ont préservé un siège au conseil d'administration pour un représentant du secteur de l'économie sociale. Il s'agit là d'un geste en apparence anodin, mais d'une importance significative au niveau de la définition d'une stratégie de développement socio-économique équilibré. En effet, les gens de l'économie sociale ont une sensibilité toute particulière face aux impacts et implications d'un dossier sur le développement global du territoire.

Toutefois, à l'instar de l'économie publique et de l'économie libérale, l'économie sociale n'a rien d'une panacée. Un territoire qui évoluerait essentiellement au rythme de l'économie sociale serait grandement handicapé. Bien que celle-ci ait le mérite de faire tourner l'économie, elle ne génère que très peu d'argent neuf, par opposition aux économies publique et libérale d'exportation ${ }^{5}$. Malgré cela, l'économie sociale ne doit pas être reléguée au rang d'économie marginale. Son rôle est certes plus effacé, mais il est néanmoins d'une importance capitale. L'économie sociale permet la réalisation de l'ensemble de ce qui ne peut, faute de rentabilité, ou ne doit, par conscience sociale, être réalisé par les deux autres secteurs de l'économie, mais qui est essentiel à un développement sain.

L'économie sociale est celle du développement à visage humain et de l'enrichissement solidaire et éthique des communautés.

Comme en toute chose, les excès et les carences sont à proscrire. Il va sans dire que c'est dans un équilibre véritable des trois facettes (libérale, publique et sociale) d'une économie globale et diversifiée qu'une région peut atteindre le but ultime, soit la résilience, c'est-à-dire la capacité d'une communauté de traverser les crises sociales et économiques en préservant 
ses acquis et en perpétuant son développement ${ }^{6}$. Dans cet esprit, l'économie sociale favorise l'augmentation de l'élasticité du tissu économique d'un territoire. Elle augmente la capacité d'une communauté à affronter les passages à vide en enracinant un certain pourcentage de l'activité économique dans des besoins immuables de la population.

Ceci dit, pour faire un parallèle avec la réalité quotidienne des développeurs régionaux, il apparaît nécessaire de mieux cibler les interventions afin d'optimiser l'utilisation des fonds. En effet, les Fonds de développement des entreprises d'économie sociale (FDEES), à ne pas confondre avec l'économie sociale au sens large, sont relativement limités et doivent être investis de façon stratégique, idéalement avec effet levier, pour faire progresser un développement cohérent et durable de la communauté. À cet effet, autant que possible, des études de marché devraient être complètes et positives, car les FDEES devraient soutenir des entreprises d'économie sociale et non des organismes proprement communautaires ou de charité. Même réalité pour ce qui est de la pertinence sociale, car ces fonds devraient être liés à un produit ou un service demandé par la communauté, mais dont la rentabilité financière n'attire pas les investisseurs privés. C'est pourquoi on nomme ce secteur économie sociale. Il ne s'agit en aucun cas de substituer l'économie sociale à l'économie libérale.

Dans le même ordre d'idées, à la suite du démarrage d'un projet, les organismes de développement devraient assurer un suivi rigoureux du dossier et s'impliquer davantage dans les premières années. Il serait également souhaitable d'encadrer les gestionnaires des entreprises en démarrage au plan de la gestion au cours des deux premières années, par exemple en intégrant dans les contrats de financement une clause de comité de gestion ou en intégrant un poste régulier au conseil d'administration.

\section{Entreprendre différemment, entreprendre collectivement}

Sur le terrain, l'économie sociale prend forme à l'intérieur d'un mode particulier de faire les choses que nous nommons entrepreneuriat collectif. Celui-ci est assimilable à l'initiative d'un groupe d'intérêt (non d'investisseur) essentiellement rassemblé pour combler un besoin clairement identifié par la communauté et, par conséquent, non satisfait par les services publics ou le secteur privé. Ainsi, les activités générées par l'entrepreneuriat collectif se constituent d'activités socio-économiques ou de démarches de dotation dans une perspective de services aux membres ou à la collectivité.

\section{La nouvelle économie sociale regroupe les entreprises qui sont apparues depuis les années 1970 et qui se sont intéressées, la plupart du temps, aux différentes facettes des conditions de vie (logement, garde d'enfants, aide à domicile, loisirs, insertion sociale et professionnelle, etc.)}

Essentiellement, l'entreprise collective se distingue de l'entreprise privée sous quatre aspects ${ }^{7}$.

1. il s'agira d'une coopérative, d'une mutuelle ou bien d'un organisme à but non lucratif;

2. il s'agira d'une entreprise caractérisée par un fonctionnement démocratique incluant la distribution des surplus et la propriété collective des excédents réinvestis;

3. il s'agira d'activités économiques de type associatif, fondées sur des valeurs de solidarité, d'autonomie et de citoyenneté, et ayant comme principe la finalité de services aux membres ou à la collectivité plutôt que le profit;

4. il s'agira d'entreprises qui peuvent inspirer un nouveau modèle de développement, qui reconnaîtrait la place et les avantages respectifs de l'économie sociale, de l'économie privée et de l'économie publique.

Par ailleurs, certaines personnes font une distinction entre les organisations dites de la nouvelle économie sociale et celles de ' $^{\text {ancienne }}{ }^{8}$. Parmi les organisations de l'ancienne définition, notons les caisses populaires, les mutuelles, les coopératives agricoles ou forestières et les commerces d'alimentation ou de quincaillerie. Créés dans un contexte bien différent, ces établissements appartiennent à l'ancienne économie sociale et tirent pratiquement la totalité de leurs revenus d'activités commerciales.

La nouvelle économie sociale regroupe les entreprises qui sont apparues depuis les années 1970 et qui se 
sont intéressées, la plupart du temps, aux différentes facettes des conditions de vie (logement, garde d'enfants, aide à domicile, loisirs, insertion sociale et professionnelle, etc. $)^{9}$. Pour notre part, la nuance apparaît intéressante dans la mesure où elle permet de distinguer différents types de solutions à différents types de besoins. Toutefois, nous n'utilisons pas systématiquement ces désignations et assimilerons à l'économie sociale l'ensemble des entreprises collectives répondant, dans le cadre des critères présentés, à une demande ou à un besoin de la communauté. Bien que, dans son rapport de 1996, le Groupe de travail sur l'économie sociale considère les organismes communautaires comme partie prenante de l'économie sociale, il propose que leur soutien et leur développement s'effectuent par le Secrétariat à l'action communautaire autonome plutôt que par les structures d'économie sociale ${ }^{10}$. Dans cet esprit, le gouvernement du Québec, en accord avec la proposition du Groupe de travail, ajoutera à sa définition les notions de viabilité et de rentabilité économique pour tracer la ligne entre l'économie sociale et le communautaire.

Dans toutes les communautés, qu'elles soient rurales ou urbaines, les besoins socio-économiques sont omniprésents. Le thème qui revient le plus fréquemment depuis quelques années est la qualité de vie. Bien qu'il s'agisse d'un concept très vague faisant parfois référence à des besoins fondamentaux (ex. : logement, nutrition) ou plus accessoires, quoique nécessaires (ex. : loisirs), beaucoup d'énergie est et sera investie pour tenter de combler ces besoins. Dans cet esprit, les intervenants du développement ont cet outil que constitue l'économie sociale pour les soutenir dans leurs tâches de développeurs régionaux. L'effet recherché à partir de cet outil est de favoriser le jaillissement d'idées novatrices, l'émergence de projets porteurs et la prise en charge par la communauté de son devenir.

\section{Nous sommes d'avis qu'il existe une voie propre à l'économie sociale : l'approche éthique}

\section{Du développement éthique et de l'éthique du développement}

Dans le cadre de cette économie plurielle dont nous avons fait état, les fonds publics sont sous le contrôle d'élus qui ont pour mission de voir à leur utilisation adéquate. Il est aussi juste d'affirmer que les capitaux privés sont soumis à une économie de marché qui, pour le meilleur ou le pire, dicte la voie à suivre aux investisseurs. Par contre, il semble que, dû à son institutionnalisation récente (Sommet sur l'économie et l'emploi de 1996), il n'existe pas de façon formelle d'aborder l'économie sociale. Certains l'aborderont comme des élus et d'autres comme des entrepreneurs privés!

Néanmoins, nous sommes d'avis qu'il existe une voie propre à l'économie sociale: l'approche éthique ${ }^{11}$. Toutefois, celle-ci nécessite d'être nuancée, car l'éthique s'applique d'une double façon : au plan du développement, que l'on qualifiera d'éthique, et au plan des relations interpersonnelles ou organisationnelles dans un processus de développement.

D'abord, le développement éthique correspond à un esprit, une philosophie de développement qui repositionne la communauté (ou à plus petite échelle la personne) comme principal objet du processus Ainsi, la communauté devient l'objet fondamental au centre de tout projet d'économie sociale. Idéalement, le développement éthique ne cherche pas le profit personnel et la croissance économique, mais le développement socio-économique de la collectivité. Toutefois, les objectifs et les résultats qui seront parfois quantitatifs, mais le plus souvent qualitatifs, n'excluent en rien les retombées économiques d'un projet. Il n'en demeure pas moins qu'un projet de croissance économique peut difficilement être directement éthique, car il vise d'abord l'enrichissement de quelques privilégiés, puis indirectement la communauté.

Un projet d'économie sociale est éthique lorsque ses objectifs fondamentaux visent l'enrichissement solidaire d'une communauté ${ }^{2}$. Dans cette perspective, le projet X comme événement en soi n'a souvent qu'une valeur relative. L'intérêt se situe au plan de son véritable gain qui est de repositionner la personne ou la communauté par le biais de son dynamisme inhérent, comme premier porteur de développement.

Ce repositionnement mènera à la pérennité (continuité de la communauté de développement) et à l'enrichissement (succession des générations d'acteurs) du processus de développement devenu processus d'auto développement. 
Bien entendu, le concept de développement éthique a parfois quelque chose de l'éthique politique, car repositionner la communauté au centre de toute décision est certes un outil fort efficace pour éviter les contestations ou en période d'élection, mais il n'en est pas moins d'une importance capitale, car structurante et efficace.

D'autre part, si le développement éthique est une philosophie, l'éthique du développement est autre chose. Il s'agit d'un outil, d'une façon d'être, d'une manière de manœuvrer au quotidien à l'intérieur d'un processus de développement. En réalité, l'éthique du développement est un art qui ne s'apprend pas. Elle s'acquiert au contact d'un territoire particulier (ex. : ville, MRC ou région administrative), car chaque territoire commande une approche éthique spécifique. Bien entendu, les réseaux d'individus transcendent les frontières (ex. : industrie agricole, industrie de transformation des métaux, etc.), mais il n'en demeure pas moins que chaque territoire se voit animé d'une logique interne spécifique.

L'éthique du développement couvre un certain nombre de détails très importants dont il faut être conscient lorsqu'on devient acteur ou promoteur du développement d'une région et qu'on se lance dans un projet d'économie sociale. Il peut s'agir des filières politiques officielles ou implicites, des groupes d'influence (gens d'affaires, professionnels, etc.), du langage (croissance, développement, ouvert, fermé), des successibilités, etc. À plus forte raison, ne pas connâ̂tre ces réalités peut mener à un échec à moyen, voire à très court terme d'un projet.

\section{Un projet de développement est éthique lorsque ses objectifs fondamentaux et les modes d'atteinte de ceux-ci visent sincèrement le bien commun et I'enrichissement collectif.}

Au minimum, un développeur devrait s'inspirer d'au moins deux des éléments fondamentaux de l'éthique du développement, soit les filières de pouvoir ou d'influence et le langage. D'une part, peut-être la partie la plus difficile, le promoteur doit parler le langage de ses interlocuteurs, adapter son discours et ses exemples afin de référer à un univers conceptuel connu de ses vis-à-vis. Il s'agit de prendre un projet relative- ment complexe de par ses tenants et aboutissants et de l'ajuster pour que l'ensemble des participants puissent bien comprendre leurs rôles et implications dans celui-ci. En définitive, le promoteur doit amener les gens à comprendre et à devenir partie prenante du projet.

D'autre part, sur le plan financier comme sur le plan technique, il sera difficile de rallier les appuis nécessaires sans avoir l'aval des personnes influentes du territoire. C'est pour cette raison que, parfois, le promoteur doit se placer en retrait pour que toute l'attention soit braquée sur une personne reconnue comme bâtisseur émérite. Pour plusieurs, ce bâtisseur constituera une sorte de cautionnement, de garantie pour la réussite de l'aventure.

En somme, un projet de développement est éthique lorsque ses objectifs fondamentaux et les modes d'atteinte de ceux-ci visent sincèrement le bien commun et l'enrichissement collectif. Parallèlement, le processus est éthique et rentable à long terme puisqu'il concerne les forces en présence et que les personnes qui incarnent ces forces ont des besoins qui se répètent, se modifient et se transforment. Certains parleront de développement durable.

$$
\mathbf{E S}=\mathbf{P}(\mathbf{e})+\mathbf{O}(\mathbf{e})
$$

ES: économie sociale P: processus O: objectifs E: éthique

Au total, le terme éthique dans « éthique du développement » a la même résonance, la même signification que le terme éthique dans "développement éthique ». Tous deux revoient la personne ou la communauté au centre des préoccupations. Par contre, si le développement éthique est principalement, mais non exclusivement, associable à l'économie sociale, il appert que l'éthique du développement touche autant l'économie publique, que l'économie libérale ou sociale.

\section{Conclusion}

En somme, il demeure que l'économie publique est encore et toujours d'une importance capitale et que l'État représente un partenaire privilégié. L'économie libérale est la locomotive du développement d'un territoire. Ceci dit, la zone d'intérêt de l'économie sociale apparaît très étendue. Par contre, après avoir mis en évidence l'importance de l'économie sociale aux côtés des économies publique et libérale, cette 
zone d'intérêt semble se préciser d'elle-même autour de besoins particuliers émanant de territoire particulier. Il appert donc que l'économie sociale est un élément essentiel de la recette d'un développement socio-économique sain et équilibré, car elle est le type d'économie le plus enraciné, le plus intégré et le plus adapté à son territoire.

En effet, l'économie sociale émane, à travers l'entrepreneuriat collectif, de la seule et unique volonté de chaque communauté d'aller de l'avant, de prendre son développement en main et surtout de déterminer collectivement les buts et objectifs à atteindre. L'économie sociale est donc ce type d'économie qui permet la réalisation de ce que seule la communauté doit réaliser, car il n'y a qu'elle qui peut se positionner en promotrice avisée et avertie de certains besoins de la communauté. Par ses contingences de démocratie et de solidarité, l'économie sociale constitue le premier lieu de gestion autonome et d'autodétermination d'une communauté.

Dans cet esprit, l'économie sociale commande une approche spécifique, car chaque territoire est unique. À cet égard, l'approche adéquate, l'approche éthique, correspond non pas à une recette miracle, mais à une philosophie qui repositionne la communauté et ses besoins comme premier objet de développement. Doublée d'une gestion de type équitable, l'approche éthique représente une voie d'accès non négligeable pour un développement sain, celui permis par l'économie sociale.

\section{Notes et références}

1 Il est à noter que toutes les coopératives ne font pas nécessairement de l'économie sociale. Certaines se comportent comme de véritables entreprises privées sans égard aux valeurs intrinsèques à l'économie sociale, tel que véhiculé aujourd'hui.

2 Inspiré de : Leclerc, Y. et C. Béland (2003), La voie citoyenne: pour renouveler le modèle québécois, Outremont, Éditions Plurimédia.

3 Définition tirée du site Internet du Chantier de l'économie sociale (définition proposée par le Chantier de l'écono- mie sociale qui a fait consensus au Sommet sur l'économie et l'emploi en octobre 1996, extrait du rapport « Osons la solidarité ») : http://www.chantier.qc.ca/.

4 Comeau, Y. (2003), Le communautaire, la nouvelle économie sociale et leurs retombées en région - ChaudièreAppalaches, L'Islet, Québec, Terres fauves.

5 Ibid. p. 132-135. Il est à noter que l'étude de M. Comeau portait essentiellement sur les entreprises de la nouvelle économie sociale, incluant les organismes communautaires. Ceci dit, nos calculs à partir des informations fournies par M. Comeau nous indiquent qu'un peu plus de $65000000 \$$ des $121492000 \$$ du budget annuel de l'économie sociale sont véritablement de l'argent neuf.

6 Selon Alain Richemond, la résilience est un terme emprunté à la physique pour exprimer l'élasticité des matériaux; élasticité qui leur permet de retrouver leur aspect initial après avoir absorbé un effort plus ou moins important. Audelà de cette limite, ils se déforment ou cassent. La résistance des matériaux aux chocs et à la pression a été, par analogie, étendue à l'homme dans sa capacité à dépasser une situation critique, à lui résister et à lui survivre. Richemond, A. (2003), La résilience économique: une chance de recommencement, Paris, Éditions d'Organisation.

7 Inspiré de Comeau, Y., op. cit.. p. 12-13.

8 Comeau, Y., op. cit.; D’Amours, M. (2000), « Procès d'institutionnalisation de l'économie sociale au Québec », Québec, Cahiers du CRISES, coll. «Working Papers », janvier, p. 1.

9 Comeau, Y., op. cit.

10 D'Amour, M., op. cit., p. 18.

11 L'éthique est un mode de pensée qui place la personne au centre. Précisément, l'éthique est un outil cognitif critique qui oriente l'action lors de situations hors normes ou non encadrées par les valeurs ou la déontologie. En d'autres mots, l'éthique fait référence à l'univers de la réflexion rationnelle et critique sur ce qui devrait orienter l'action.

12 À ne pas mettre en opposition avec l'économie libérale qui peut viser aussi l'enrichissement de la communauté. Toutefois, l'économie libérale vise l'enrichissement de la communauté comme effet secondaire de l'enrichissement du promoteur. 